\title{
Enhancement of Tera-Hertz Radiation by carrier dynamics modulation with chirped optical pulses
}

\author{
Chao-Kuei Lee ${ }^{1}$, Chung-Han Wu ${ }^{2}$, Chin-Rong Chung ${ }^{2}$, and Ci-Ling Pan $^{2}$ \\ ${ }^{1}$ Institute of Electro-Optical Engineering, National Sun Yat Sen University, Taiwan, ROC \\ ${ }^{2}$ Department of Photonics and Institute of Optoelectronic Engineering, National Chiao Tung University, Taiwan, \\ ROC
}

\begin{abstract}
In this article, enhancement of $\mathrm{THz}$ radiation was studied using pulse shaping thchnique. Over $40 \%$ enhance was demonstrated by positive chirped optical pulses. This could be attributed to the pump-dump process in excitation.
\end{abstract}

Keywords: THz radiation, ultrafast, carrier dynamics, chirped pulses

\section{INTRODUCTION}

$\mathrm{THz}$ free-space radiation has been used in applications in coherent time-domain spectroscopy, and in imaging and ranging of objects. ${ }^{1,2}$ For this reason a number of researchers have investigated the optimum design of photoconductive $\mathrm{THz}$ transmitters and the physical mechanisms that limit the radiation power. ${ }^{3-5}$ Past, enhancement of narrow-band terahertz radiation using optical pulse shaping was demonstrated. ${ }^{6}$ In addition, frequency tunable terahertz radiation was also reported. In this article, enhancement of $\mathrm{THz}$ radiation was studied using pulse shaping technique. Over $40 \%$ enhance was demonstrated by chirped optical pulses. This could be attributed to the carrier lifetime modulation resulted from pump-dump process in excitation

\section{Experimental Setup}

Fig.1 shows the experimental setup of our adaptive controlled $\mathrm{THz}$ radiation system. A mode-locked Ti:sapphire laser provided excitation laser pulses of approximately $40 \mathrm{fs}$ at a repetition rate of $82 \mathrm{MHz}$. The pulses passed through a grating and mirror optical pulse shaper. In our adaptive feedback pulse shaping system, new freezing algorism was used in optimization. ${ }^{7}$ After calculating the adaptive phase by SLM, chirped condition could be obtained.

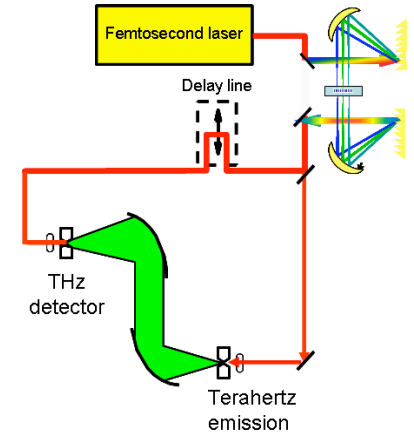

Fig. 1. Experimental setup of adaptive controlled $\mathrm{THz}$ radiation system

\section{Results and discussion}

Figure 2 shows temporal profile and corresponding spectrum of terahertz radiation under various laser conditions. As shown in up-plot of Fig. 2, similar temporal and spectral profiles were observed. The triangle one (case of chirp-free) is the case of which the pulse before terahertz generation system is almost transform-limited modulated by pulse shaper. The central frequency of the radiations is around at $0.3 \mathrm{THz}$. Smaller radiation intensity was obtained under case of which the pulse incident on antenna is chirped free, see case of antenna free. This indicates that transformlimited pulse on antenna is not the best choice in generating more radiation. In addition, positive chirp pulse from optical components could be favor in enhancement of terahertz radiation. To further clarify, terahertz signal was feedback to optimize through new freezing algorism. As shown the blue cross curve (case of $\mathrm{THz}$ free) in fig. 2, larger radiation was demonstrated. Over $40 \%$ enhancement was observed, compared to the case of chirped free pulse incident on antenna.

Figure 3 shows the phase distribution in frequency domain. Positively chirped phase was retrieved from the curve. In the past, increasing radiation as decreasing carrier was reported. ${ }^{8}$ For example, positively chirped excitation will result in band gap 
renormalization or band filling. ${ }^{9}$ It is well known that band filling will reduce carrier lifetime of material. Therefore, the enhancement could be attributed to the carrier lifetime modulation resulted from pump-dump process in excitation.
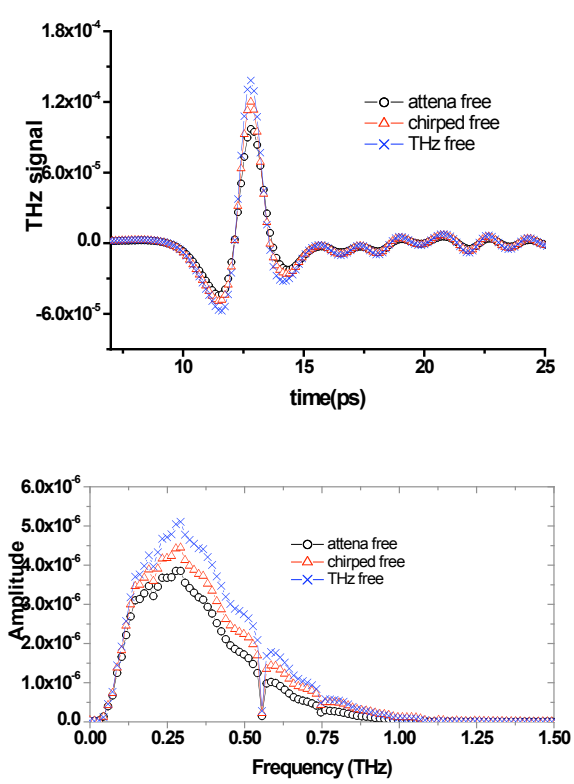

Fig. 2. Up: Temporal profile of $\mathrm{THz}$ radiation of various conditions: attena free, chirped free and $\mathrm{THz}$ free; Down:

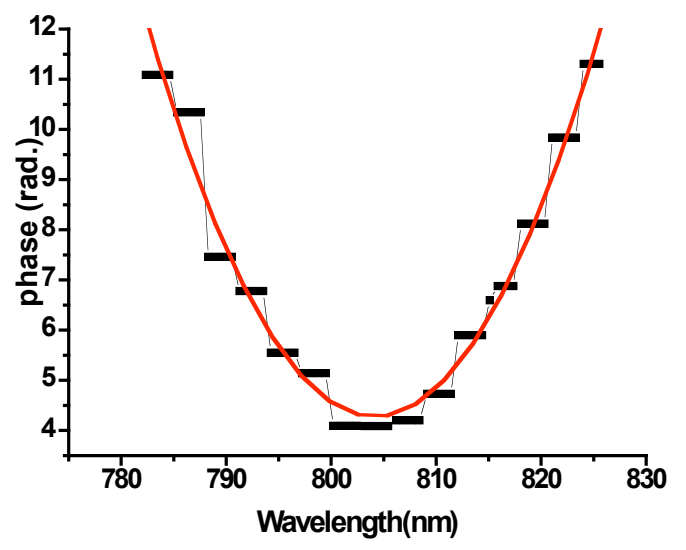

Fig. 3. Phase profile of laser for $\mathrm{THz}$ enhancement

\section{Conclusion}

Enhancement of $\mathrm{THz}$ radiation was studied using pulse shaping thchnique. Over $40 \%$ enhance was demonstrated by chirped optical pulses. This could be attributed to the carrier lifetime modulation resulted from pump-dump process in excitation

\section{Acknowledgment}

This work was supported in part by the Academic Excellence Program of the Ministry of Education under contract No. (91-E-FA08-1-4), and by the National Science Council under contract No. (NSC93-2218-E-110-040), Taipei, Taiwan, ROC. Authors also would like to thank Prof. M. L. Hsieh of NTNU in calibration of spatial light modulation.

\section{References}

[1] B. B. Hu and M. C. Nuss, Opt. Lett. 20, 1716 (1995)

[2] R. A. Cheville and D. Grischkowsky, Appl. Phys. Lett. 67, 1960 (1995).

[3] W. Sha, J. Rhee, T. Norris, and W. J. Schaff, IEEE J. Quantum Electron. 28, 2445 (1992).

[4] I. Brener, P. C. M. Planken, M. C. Nuss, M. S. C. Luo, S. L. Chuang, L. Pfeiffer, D. E. Leaird, and A. M. Weiner, J. Opt. Soc. Am. B 11, 2457 (1994).

[5] J. T. Darrow, X.-C. Zhang, D. H. Auston, and J. D. Morse, IEEE J. Quantum Electron. 28, 1607 (1992).

[6] P. K. Benicewicz, J. P. Roberts, and A. J. Taylor, J. Opt. Soc. Am. B 11, 2533 (1994).

[7] B. I. Greene, J. F. Federici, D. R. Dykaar, R. R. Jones, and P. H. Bucksbaum, Appl. Phys. Lett. 59, 893 (1991).

[8] Yongqian Liu, Sang-Gyu Park, and A. M. Weiner, Opt. Lett. 12, 1762 (1996).

[9] A. M. Weiner, D. E. Leaird, J. S. Patel, and J. R. Wullert, IEEE J. Quantum Electron. 28, 908 (1992).

[10] Z. S. Piao, M. Tani, and K. Sakai, Jpn J. Appl. Phys, 39, 96(1999)

[11] D. F. Liu, and J.Y. Qin, Appl. Opts. 42, 3679(2003)
- 